\title{
POLYNOMIAL INVERSE IMAGES AND POLYNOMIAL NUMERICAL HULLS OF NORMAL MATRICES
}

\author{
Hamid Reza Afshin, Mohammad Ali Mehrjoofard \\ AND ABBAS SALEMI
}

Abstract. Let $A \in M_{n}$ be a normal matrix and let $k \in \mathbb{N}$. In this note we introduce the notion "Polynomial inverse image of order $\mathrm{k}$ ". The polynomial numerical hull of order $k$, denoted by $V^{k}(A)$ are characterized by the intersection of polynomial inverse images of order k. Also, the locus of $V^{n-1}(A)$ in the complex plane are determined.

Mathematics subject classification (2010): 15A60, 15A18, 14H45, 30C15, 52A10.

Keywords and phrases: Polynomial numerical hull, polynomial inverse image, joint numerical range, normal matrices.

\section{REFERENCES}

[1] H. R. Afshin, M. A. Mehrjoofard And A. Salemi, Polynomial numerical hulls of order 3, Electronic Journal of Linear Algebra, 18 (2009), 253-263.

[2] Ch. Davis, C. K. Li And A. S Alemi, Polynomial numerical hulls of matrices, Linear Algebra and its Applications, 428 (2008), 137-153.

[3] CH. DaVis AND A. SAlEmi, On polynomial numerical hulls of normal matrices, Linear Algebra and its Applications, 383 (2004), 151-161.

[4] V. FAber, W. Joubert, M. Knill and T. Manteuffel, Minimal residual method stronger than polynomial preconditioning, SIAM Journal on Matrix Analysis and Applications, 17 (1996), 707-729.

[5] A. Greenbaum, Generalizations of the field of values useful in the study of polynomial functions of a matrix, Linear Algebra and Its Applications, 347 (2002), 233-249.

[6] B. Grünbaum, Convex Polytopes, John Wiley and Sons, New York, 1967.

[7] O. Nevanlinna, Convergence of Iterations for Linear Equations, Birkhäuser, Basel, 1993.

[8] O. Nevanlinna, Hessenberg matrices in Krylov subspaces and the computation of the spectrum, Numerical Functional Analysis and Optimization, 16 (1995), 443-473. 